\title{
Effect of structural disorder on electronic states in GaAs/AIGaAs quantum wires
}

\author{
Jasprit Singh \\ Department of Electrical Engineering and Computer Science, The University of Michigan, Ann Arbor, \\ Michigan 48109
}

(Received 26 June 1991; accepted for publication 10 September 1991)

\begin{abstract}
Perfect quantum wire structures are attractive candidates for low threshold lasers and high speed electronic devices because of the nature of the density of states and eigenfunctions. In this letter, we discuss the effect of structural disorder on the density of states as well as on the localization length of these eigenstates. We find that significant changes in the density of states and eigenfunctions occur with a small random disorder along the wire axis. Consequences for devices based on quantum wires are discussed.
\end{abstract}

Quasi-one-dimensional systems are currently attracting a great deal of interest for their potential applications in high speed electron devices and extremely low threshold current lasers. ${ }^{1-4}$ Much of this potential is due to the nature of the one-dimensional (1D) band structure, the density of states and the reduced phase space for scattering. The density of states develop a strong peak near the band edge for quantum wires, allowing for example a very high gain in quantum wire lasers at very low injection. The ideal 1D density of states is ${ }^{5}$

$$
N(E)=\frac{1}{\sqrt{2} \pi \hbar}\left(E-E_{n}\right)^{-1 / 2},
$$

where $E_{n}$ is the confinement energy due to the finite cross section of the wire. The wave functions along the axis of the wire ( $x$-axis) are described by Bloch functions

$$
\Psi(x)=\frac{1}{\sqrt{l}} U_{v k} e^{i k x}
$$

if the wire is perfect. Here $U_{v k}$ is the central cell part of the Bloch function which in the effective mass theory may be taken as the bulk materials band edge central cell function. As noted above, the density of states and the wave function are strictly valid only for the perfect wire. In fact, in a one-dimensional system, we know that any disorder can localize states and drastically alter the density of states. ${ }^{5}$ Since most of the predicted benefits of quantum wires arise from these special features, it is important to find out whether in realistic quantum wires which are not strictly one dimensional, but are quasi-one-dimensional (due to the finite wire cross section), structural disorder will drastically alter the electronic properties.

It is important to distinguish between the fabrication process of quantum wells versus quantum wires. The quantum wells are formed by a simple shuttering mechanism which leads to an interfacial structure fluctuation of the order of only a monolayer $(\sim 3 \AA)$ or so. No such simple process exists for quantum wire fabrication. While a number of different techniques have been employed for quantum wires, it is expected that it would be very difficult to approach the perfection of quantum wells, and a structural variation of less than $10-20 \AA$ may be difficult to achieve.
With this in mind, we consider a quantum wire as shown in Fig. 1. The wire has a cross section of $L_{y} L_{z}$ and has a sidewall of disordered region with width $\Delta L_{z}$. The disordered sidewall is made up of islands of either the barrier region (which we choose to be $\mathrm{Al}_{0.3} \mathrm{Ga}_{0.7} \mathrm{As}$ ) or $\mathrm{GaAs}$ (which is the well region material). These islands are randomly placed on this edge by a Monte Carlo method. In the results shown for the disordered cases, we have assumed a 50:50 chance of the islands on the disordered edge being $\mathrm{GaAs}$ or $\mathrm{Al}_{0.3} \mathrm{Ga}_{0.7} \mathrm{As}$. $\mathrm{A}$ number of other kinds of disorder (e.g., along two edges) were also introduced, but the results were qualitatively the same.

A $2000 \AA$ long wire was used for the results that will be presented. A finite size wire had to be chosen because we solve the Schrödinger equation in the disordered wire by the finite difference method which effectively involves the diagonalization of a $1000 \times 1000$ matrix. We find that with a $2000 \AA$ wire, the density of states results converge to the infinite wire results. A $2 \mathrm{meV}$ Gaussian broadening is used for the density of states results presented here.

In Fig. 2 we show the density of states results for the perfect wire as calculated by the numerical technique. A
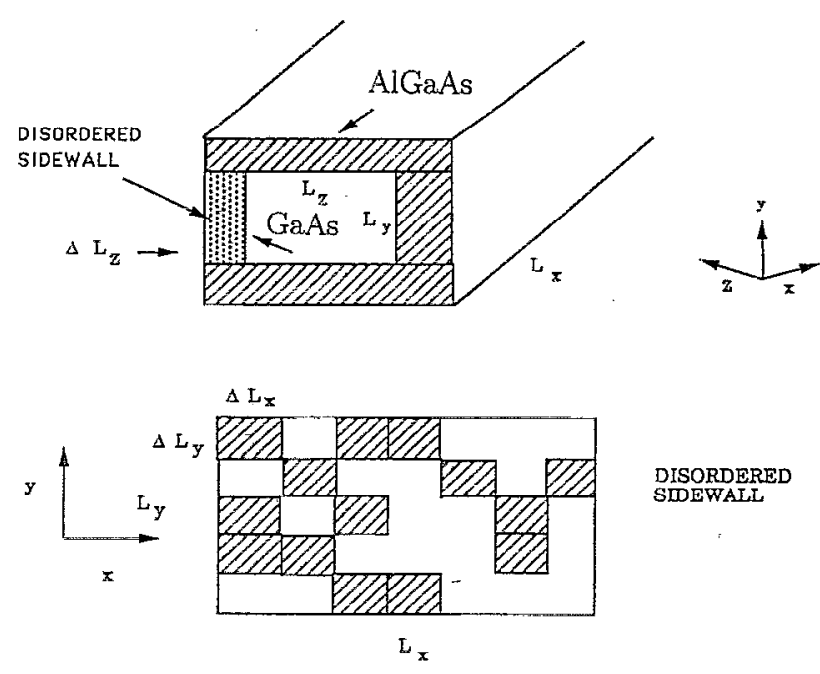

FIG. 1. A schematic of the model used to introduce structural disorder along the quantum wire axis. Random islands having composition of either well or barrier material and sizes $\Delta L_{x} \times \Delta L_{y} \times \Delta L_{z}$ are placed along one of the sidewalls. 
$60 \times 60 \AA$ wire size was used with an $\mathrm{Al}_{0.3} \mathrm{Ga}_{0.7} \mathrm{As}$ barrier. Figure 2(a) shows the density of states and we can see the sharp peak at the effective band edge (measured from the bulk conduction band edge). The results are shown for the ground state of the confined state in the $y-z$ plane. The excited states results are at a much higher energy and are not shown. We only focus on an energy region of about $30-40 \mathrm{meV}$ away from the band edge since this is the region of interest for many devices. The stationary wave functions are represented by plotting the probability function $|\Psi(x)|^{2}$ along the wire axis. The electron probability function has a peak amplitude of 0.05 corresponding to the normalization in the $0.2 \mu \mathrm{m}$ wire.

In Fig. 3, we show the effect of disorder on the quantum wire electronic properties. The disorder is introduced by randomly placing islands along one edge of the wire (see Fig. 1) with island sizes given by

$$
\Delta L_{z}=20 \AA ; \quad \Delta L_{j}=20 \AA ; \quad \Delta L_{x}=100 \AA .
$$

The inner $60 \times 60 \AA$ GaAs region is still perfect, as are the three other edges of the wire. Thus the disorder introduced is not of an extreme nature. The random potential fluctuations (averaged over the islands in the $z$ direction) seen by the electron along the wired axis are shown in Fig.

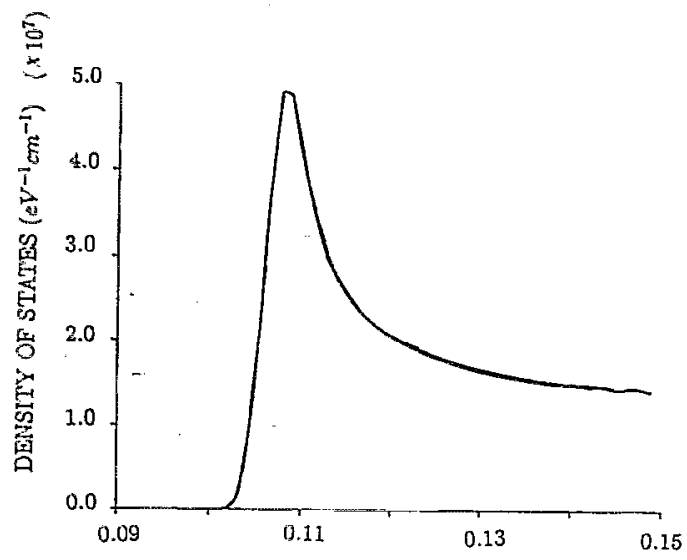

(a) ENERGY (eV)

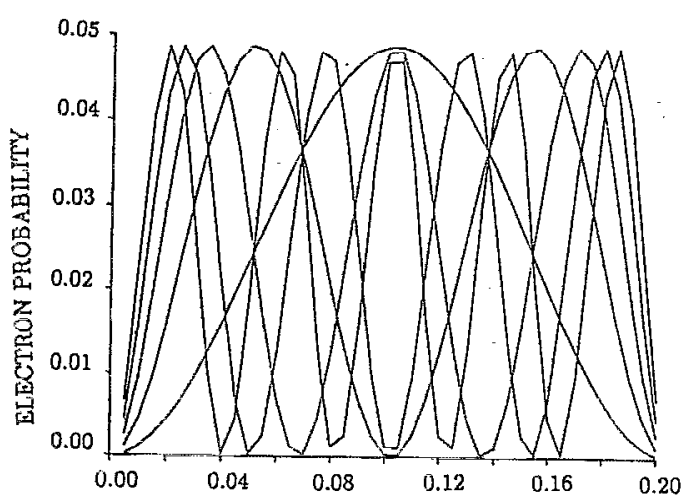

(b)

DISTANCE ALONG WIRE AXIS ( $\mu \mathrm{m})$

FIG. 2. (a) Calculated density of states (including $2 \mathrm{meV}$ Gaussian linewidth) for the perfect wire and (b) probability distribution for some of the low lying states. 3(a). The density of states obtained by diagonalizing the effective mass matrix with the disordered potential is shown in Fig. 3(b). The density of states is not too different from the case of the perfect wire [Fig. 2(a)]. There is only a $10 \%$ reduction in the peak value at the band edge and the density of states has some other peaks. These other peaks shift around if the disorder is generated in a different random sequence. However, the main peak at the band edge is maintained in spite of the disorder. This is in contrast to the case of a strictly 1D system where the disorder qualitatively alters the density of states. ${ }^{5}$

The nature of the wave functions in the disordered wire however is dramatically affected by the disorder. The probability functions for the first five lowest energy states are shown in Fig. 4(a). Once again $|\Psi(x)|^{2}$ is plotted as a function of the position along the wire axis. The electronic states are all strongly localized in a particular region of the wire axis. The lateral spread ( localization length) of these states is $\sim 300-400 \AA$. Once again if we charge the random sequence of the disorder, the ordering of these states does change, but the localization length is in the same range. As we move to higher energy states, the extent of the states increases slowly. In Fig. 4(b), we show a state $\sim 20 \mathrm{meV}$ above the lowest state. As can be seen, this state

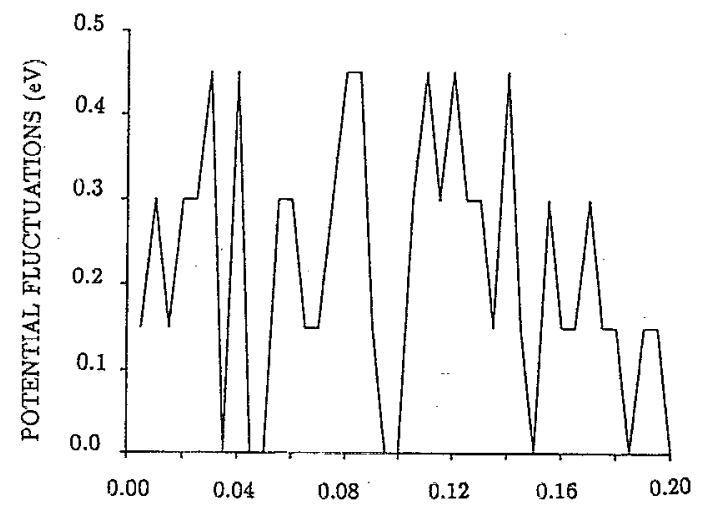

(a) DISTANCE ALONG WIRE AXIS $(\mu \mathrm{m})$

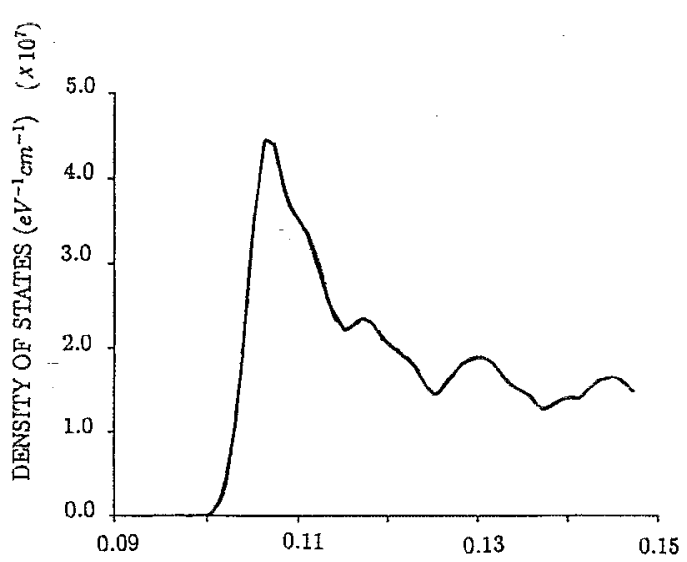

(b)

ENERGY (eV)

FIG. 3. (a) Random potential seen along the $20 \AA$ sidewall region along the quantum wire axis, $(b)$ density of states for the disordered quantum wire. 


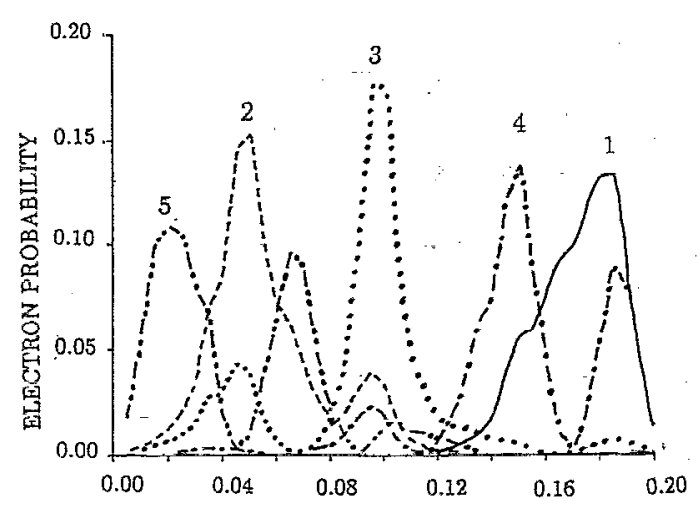

(a)

DISTANCE ALONG WIRE AXIS ( $\mu \mathrm{m})$

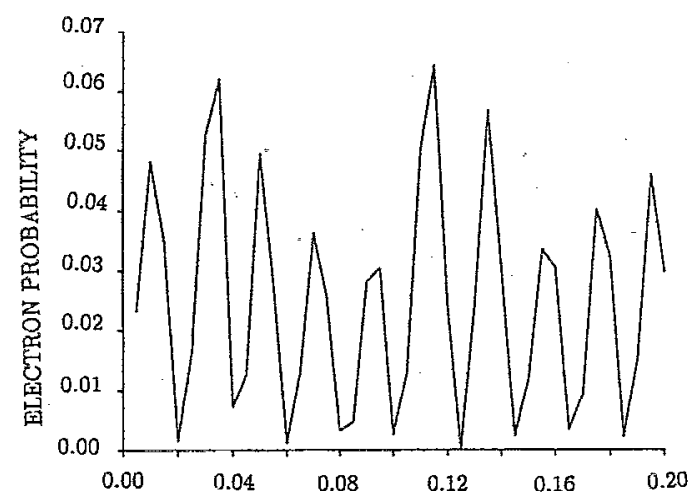

(b)

DISTANCE ALONG WIRE AXIS $(\mu \mathrm{m})$

FIG. 4. (a) Probability function for the lowest lying five states along the wire axis for the disordered wire, (b) probability function for a state 20 $\mathrm{meV}$ above the lowest lying state. is extended over the entire range of the wire and looks similar to the excited states of the perfect wire.

The calculations were repeated for the heavy hole states and it was found that in this case, while the density of states once again maintained a peak at the band edge, all the states up to $25 \mathrm{meV}$ were strongly localized. The localization lengths were $\sim 100-200 \AA$ for the lower lying states. The fluctuations were therefore much more effective in localizing the heavier hole.

Our simulation results show that small disorder does not qualitatively perturb the nature of the density of states function, although it causes localization of a large fraction of the low-lying electronic states. Properties of the quantum wire which are more dependent upon the density of states such as optical transitions will still see an enhancement due to the peak at the band edge in the density of states. However, the usual $k$-selection rules for optical transitions may not be valid for these calculations. Properties of the quantum wire which are dependent upon the extended nature of the wave function such as charge transport, are expected to be significantly altered. This study shows the seriousness of the effect of disorder on the wire properties. Detailed studies with varying levels of disorder will be presented in a longer paper.

This work was partially supported by the United States Army's URI program. (Grant No. DAAL03-87-K-0007.)

${ }^{1}$ Y. Arakawa and H. Sakaki, Appl. Phys. Lett. 40, 939 (1982).

${ }^{2}$ Y. Arakawa, K. Vahala, and A. Yariv, Appl. Phys. Lett. 45, 950 (1984).

${ }^{3}$ H. Sakakí, Jpn. J. Appl. Phys. 19, L735 (1980).

${ }^{4}$ See for example, E. N. Economou, Green's Functions in Quantum Physics (Springer, New York, 1979).

${ }^{5}$ See for example, E. N. Economou, Green's Functions in Quantum Physics (Springer, New York, 1979), Chap. 7. 